\title{
Textural Analysis of Spinous Layer for Grading Oral Submucous Fibrosis
}

\author{
Rusha Patra \\ Department of Electrical \\ Engineering \\ Indian Institute of Technology \\ Kharagpur
}

\author{
Chandan Chakraborty \\ School of Medical Science and \\ Technology \\ Indian Institute of Technology \\ Kharagpur
}

\author{
Jyotirmoy Chatterjee \\ School of Medical Science and \\ Technology \\ Indian Institute of Technology \\ Kharagpur
}

\begin{abstract}
Spinous layer being a sub compartment of surface epithelium of oral mucosa, plays also major role in investigating oral submucous fibrosis (OSF) at its early stage in addition to basal layer. This paper aims to provide a technique that can be used to assist the oral pathologists in grading OSF based on textural information of the spinous layer. The proposed scheme intends to evaluate the textural changes from normal to various grades of OSF. In practice, it comprises the following modules - (a) surface epithelium segmentation, (b) selection of windows on the spinous layer in reference to the basal layer, (c) textural feature extraction and analysis and finally (d) grading. Here the epithelium is segmented using anisotropic diffusion and Otsu's thresholding. Wavelet based multi-resolution technique is applied to extract 12 textural features from spinous layer. From the statistical analysis, it is observed that 6 features are significant in discriminating normal, OSF with and without dysplasia. Finally, support vector machine (SVM) and Bayesian classifiers are trained with 46 normal, 24 OSF without dysplasia and 20 OSF with dysplasia samples for OSF grading. The result shows that the classification accuracies for both the classifiers (Bayesian = $93.3 \%, \mathrm{SVM}=96.6 \%$ ) are comparable, there by emphasizing the significance of texture in oral cancer diagnostics.
\end{abstract}

\section{General Terms}

Image processing, Computer Aided Diagnosis system.

\section{Keywords}

Oral submucous fibrosis (OSF), spinous layer, anisotropic diffusion, wavelet, statistical test, Bayesian classifier, support vector machine.

\section{INTRODUCTION}

Medical history has been witnessed for increasing oral cancer over recent years and more than 0.3 million new cases of oral cancer are reported each year [1]. A high incidence of oral cancer is mainly due to the late diagnosis of potential precancerous lesions and conditions [2]. Oral submucous fibrosis (OSF) is an insidious chronic progressive precancerous condition of the oral cavity [3] and a large proportion of this precancerous lesion converts to squamous cell carcinoma. Histopathologically OSF is characterized by the concomitant presence of less vascularized collagenous connective tissue with overlying atrophic epithelium in oral mucosa [4].

Oral epithelial dysplasia is the diagnostic term used to describe the histopathologic changes seen in a chronic, progressive and premalignant disorder of oral mucosa. In these cases, the epithelial cells show an altered maturation pattern and cytological changes such as cellular and nuclear pleomorphism, nuclear hyperchromatism, increased nuclear/cytoplasmic ratio, prominent nucleoli, increased mitotic activity, increased intercellular space, loss of epithelium cell cohesion and so on [5]. Due to these changes, the overall architecture of the epithelium has changed. Traditionally, the pathologists use histopathological images of biopsy tissue and examined them under light microscope to detect OSF which is a highly qualitative process. Landini and Rippin were the first who tried to overcome the subjectivity in diagnosing the oral malignancy in 1996. They have proposed histopathological diagnosis of oral premalignancy and malignancy based on the morphological characteristics of the cells and tissues in 2-D section of histopathological images [6]. In 2004, Landini and Othman have proposed an automatic method based on morphological reconstruction to describe the hierarchical architectural characteristics of surface epithelium for oral cancer, dysplastic and healthy conditions [7]. The severity of the atypical changes (cellular, nuclear pleomorphism, tear-drop shaped rete-ridges) and the height in the epithelium to which these changes extend have been studied for the grading of dysplasia [8]. Beyond tissue component features (classically the cell and nuclear morphology), the structural characteristics of tissue (e.g. complexity of tissue profile) has been described, but limited work has been done on statistical description of tissue spatial architecture [9-10]. The textural analysis has been mostly done on sub-epithelial connective tissue (SECT). First attempt has been made to analyze the transmission electron microscopic (TEM) images of sub-epithelial collagen fibers in early and advance stages of OSF using wavelet and neural network based technique [11]. Different wavelet features have been studied to classify progressive stages of OSF [12]. Cells in SECT region are also studied and automated classification of SECT cells using SVM based approach have been performed [13]. In the literature, it has been observed that the classifier based on textural analysis of epithelial tissue for the detection of OSF has been attempted less [14]. It can be noted that the oral cancer initiates from the basal layer, so it is very much important to explore the basal layer as well as the other sub-layers of oral mucosa for studying related morphological and textural features.

In view of this, the present paper focuses on the quantification of the change of textural aspect of epithelium for the characterization of normal and different grades of OSF. To quantify the overall texture of the spinous or prickle layer cell, the wavelet and subband decomposition of the selected ROI (region of interest) has been performed followed by the classification of the normal and different grades of OSF. To improve the classification accuracy, the statistical analysis of 
the features has been prepared to facilitate feature selection. This work aims to help the oral pathologists in screening of oral precancer with the intention of minimizing the subjectivity in the present light microscopic qualitative assessment procedure.

\section{MATERIALS AND METHODS}

All normal and oral submucous fibrosis (OSF) biopsy samples are collected from Department of Oral and Maxillofacial Pathology, Guru Nanak Institute of Dental Sciences and Research, Kolkata, India. Those biopsy samples were processed for histopathological examination and paraffin embedded tissue sections of $5 \mu \mathrm{m}$ thickness were prepared and then stained by Haematoxylin and Eosin (H \& E). 50 images of normal oral mucosa, 30 images of OSF without dysplasia and 20 images of OSF with dysplasia have been considered in this study. The images are captured using a light microscope mounted with an Axio Vision camera attached to a Zeiss Observer.Z1 Microscope (CarlZeiss, Germany) system. Lowpower magnification of 40x (numerical aperture 0.6) was used for grabbing the images. All the images are sampled and digitized to 24 bits of color information and stored as $1388 \times 1040$ pixel with resolution of $0.16 \mu \mathrm{m}$.

The proposed methodology includes anisotropic diffusion based segmentation, feature extraction by wavelet decomposition followed by classification by using Bayesian classifier and Support Vector Machine (SVM). Practically, surface epithelium of oral mucosa has been segmented out by using anisotropic diffusion and thresholding. Then ROI (a window of $128 \times 128$ pixel) is selected in the spinous layer of the surface epithelium. Textural features are extracted by using wavelet decomposition of the ROI. Features' distribution and significance are estimated by using JarqueBera test, boxplot, one-way ANOVA (Analysis of Variance). Finally the selected features are fed to the Bayesian and SVM classifier to characterize the normal, OSF without dysplasia and OSF with dysplasia. In addition, the diagnostic performance of the classifiers is evaluated. The methodology is summarized below using a schematic diagram as in Figure 1.

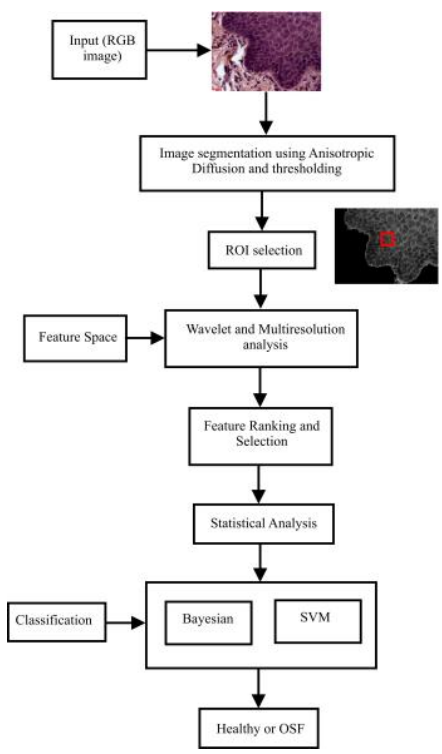

Figure 1: Block diagram of the proposed methodology for Screening of OSF

\subsection{Anisotropic diffusion}

Anisotropic diffusion is a technique aiming at removing or smoothing of the homogeneous part of an image while keeping the significant part of the image like edge, line and other details that are important for the image interpretation. It encourages intra region smoothing in preference to inter region smoothing so that the edge or the boundary between two regions is preserved [15]. The image is diffused for 100 iterations. In the diffused image, the edges or boundaries are made sharp and Otsu's thresholding is done to segment out the surface epithelium.

A window of size $128 \times 128$ pixels is selected as described below. A random number is generated and it is checked whether the point is in surface epithelium or not. If the point is within the epithelium, then its distance from each point on the boundary is calculated. The point would be considered as a point in spinous layer if the minimum distance is less than a threshold value. Biologically, the lowermost layer of the surface epithelium is basal layer which is a single layer of basal cells and the layer just above the basal layer is spinous layer. Spinous layer is stratified having 3-4 layers of cells [16]. From the size of the cells and image resolution, it has been observed that the basal layer can be extracted by morphological erosion and subtraction using a disk structuring element of radius 100 pixels. Considering 3 layers of cells in spinous layer, the threshold value is decided as 800 pixels. Subsequent to the selection of the point, a window is selected taking the selected point as the top left corner of the window. Textural analysis has been done on this window using wavelet based multiresolution analysis.

\subsection{Wavelet based multiresolution analysis}

Wavelets are a class of functions used to localize a given function in both space and scale. The concept of multiresolution analysis, invented by Mallat [17], can be viewed as a successive approximation or refinement of a signal. At each step in decomposition procedure, the signal is decomposed into four independent channels, producing four subimages. At each step of decomposition, the low frequency component (coarse approximation subimage) of the previous step is decomposed again. The images are decomposed by HAAR or DB1 wavelet into three levels. In each level, the detail and approximate coefficients are extracted. Since the image size is $128 \times 128$, the dimension of the coefficients at $i^{\text {th }}$ level is $128 * 2^{-i} \times 128 * 2^{-i}$ for $\mathrm{i}=1,2,3$. The frobenious norm has been computed for all the coefficients at each level. The elements of the feature vector are the frobenious norm of the approximate and detail coefficients of each level.

\subsection{Statistical analysis of the textural features}

Prior to classification, it is essential to verify whether the features have adequate discriminating potential to characterize the labeled classes; normal, OSF without dysplasia and OSF with dysplasia.

The significance of the features is tested with the help of oneway analysis of variance (ANOVA) [18]. To test the null hypothesis that the population means of all the groups or classes are equal against the alternative hypothesis that all the means are not equal; ANOVA produces an F statistic, a higher ratio of which implies that the samples were drawn from different population. 
Jarque-Bera test is a goodness-of-fit measure of departure from normality. Based on the sample kurtosis and skewness, the test statistic is

$$
J B=\frac{n}{6}\left(S^{2}+\frac{1}{4} K^{2}\right)
$$

where $\mathrm{n}$ is the sample size, $\mathrm{S}$ is the sample skewness and $\mathrm{K}$ is the sample kurtosis. The statistic JB follows an asymptotic Chi-square distribution with two degrees of freedom to test the null hypothesis that the data are from a normal or Gaussian distribution against the alternative hypothesis that the population distribution is not normal [19]. A high value of test statistic indicates that the samples are not from a normal distribution. be justified, not ragged.

\subsection{Feature selection}

Feature selection has been performed to utilize the classifier at its fullest ability. The higher $F$ value of a feature implies that the discriminating potential of that feature is more than the others. So depending on the F values, obtained from the oneway ANOVA, the features are ranked such that more the F value, the rank will be higher. The features having high rank and also following Normal distribution are selected to be fed to the classifier.

\subsection{Statistical classification}

\subsubsection{Bayesian classifier}

Bayesian classification is based on Baye's decision theory of probability. It basically minimizes the misclassification errors substantially and chooses the most probable option [20]. Let there are $\mathrm{n}$ features having feature space, and $\omega_{i}$ is the $i^{\text {th }}$ class. The sample will be belonging to $i^{\text {th }}$ class, if

$$
P\left(\omega_{i}\right) p\left(x / \omega_{i}\right)>P\left(\omega_{j}\right) p\left(x / \omega_{j}\right) \forall j \neq i
$$

where $P\left(\omega_{i}\right)$ is prior and $p\left(x / \omega_{i}\right)$ is likelyhood.

\subsubsection{Support vector machine}

SVM is a statistical learning network which maximizes the distance between the class and separating hyper-plane [21]. The discriminant function is such that it puts two classes on the either side of the plane. The width of the separating hyperplane has to be maximized [22].

Since it is a three class problem, first it determines whether the test sample is normal or diseased and then if it is diseased then it will determine the grade of the disease i.e. OSF without dysplasia or with dysplasia.

\subsection{Performance analysis}

Cross validation Cross validation approach is a technique for assessing how the results of a statistical analysis will generalize to an independent data set. Cross validation involves partitioning a sample of data into complimentary subsets, performing the analysis on one subset and validating the analysis on the other subset. Multiple rounds of cross validation are performed using different partitions to reduce variability, and the validation results are averaged over the rounds. The different partitions, which have been used here, are given below.

- First $3 / 4^{\text {th }}$ data used for training and last $1 / 4^{\text {th }}$ data used for testing.
- Last $3 / 4^{\text {th }}$ data used for training and first $1 / 4^{\text {th }}$ data used for testing.

- First $1 / 8^{\text {th }}$ data used for testing, after that $3 / 4^{\text {th }}$ data used for training and last $1 / 8^{\text {th }}$ data used for testing.

Therefore the outcomes of these three trials are averaged and the mean values for different classifiers are compared.

The performance evaluation of the classifiers has been carried out on the basis of individual accuracy and overall accuracy. Individual accuracy is the proportion of correctly classified samples in a particular class and overall accuracy is the proportion of correctly classified samples for all the classes.

\section{RESULTS AND DISCUSSIONS}

The surface epithelium is segmented out in normal and OSF without and with dysplasia images by using anisotropic diffusion followed by thresholding and morphological operations (opening, filling). The results are shown in Figure 2 and the selected ROIs (marked as a red box) are shown in Figure 3. The number of iterations in anisotropic filtering is fixed to 100 for all the images. The value is set heuristically but it is same for the whole image database. The top left corner of the ROIs are selected by generating a random number which satisfies the predefined conditions. The random numbers are generated by the random number generation command of MATLAB and hence it can be assumed that there is no bias in selection process as it is independent of any expert's selection. The textural analysis has been done on the selected ROIs.
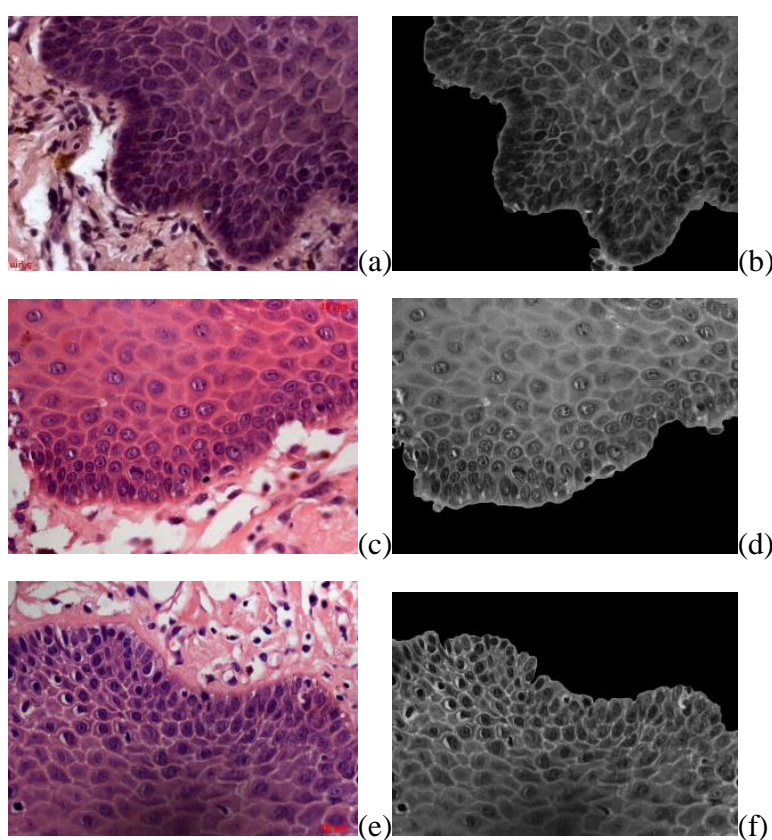

Figure 2: (a)Normal Oral Mucosa, (b)Segmented Surface Epithelium of (a), (c)OSF without dysplasia, (d) Segmented Surface Epithelium of (c), (e)OSF with dysplasia, (f) Segmented Surface Epithelium of (e)

The descriptive statistics of the features, as mentioned above, are given in Table 1 and also the boxplot for all the features are computed, six of them (the selected features) are shown in Figure 4. It is apparent from Table I that for some of the features, the mean value of different classes is distinctly different whereas for some of them they are nearly same. 

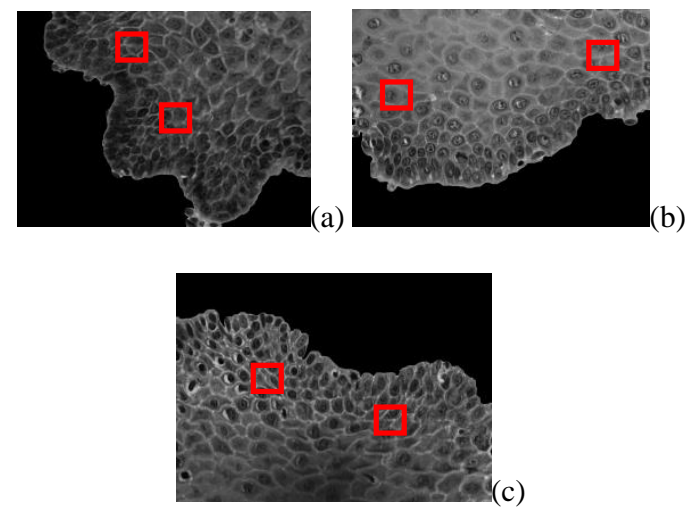

Figure 3: Gray scale images showing windows (red coloured square) for (a) normal, (b) OSF without dysplasia and (c) OSF with dysplasia

Table 1. Descriptive Statistics of the Feature Space

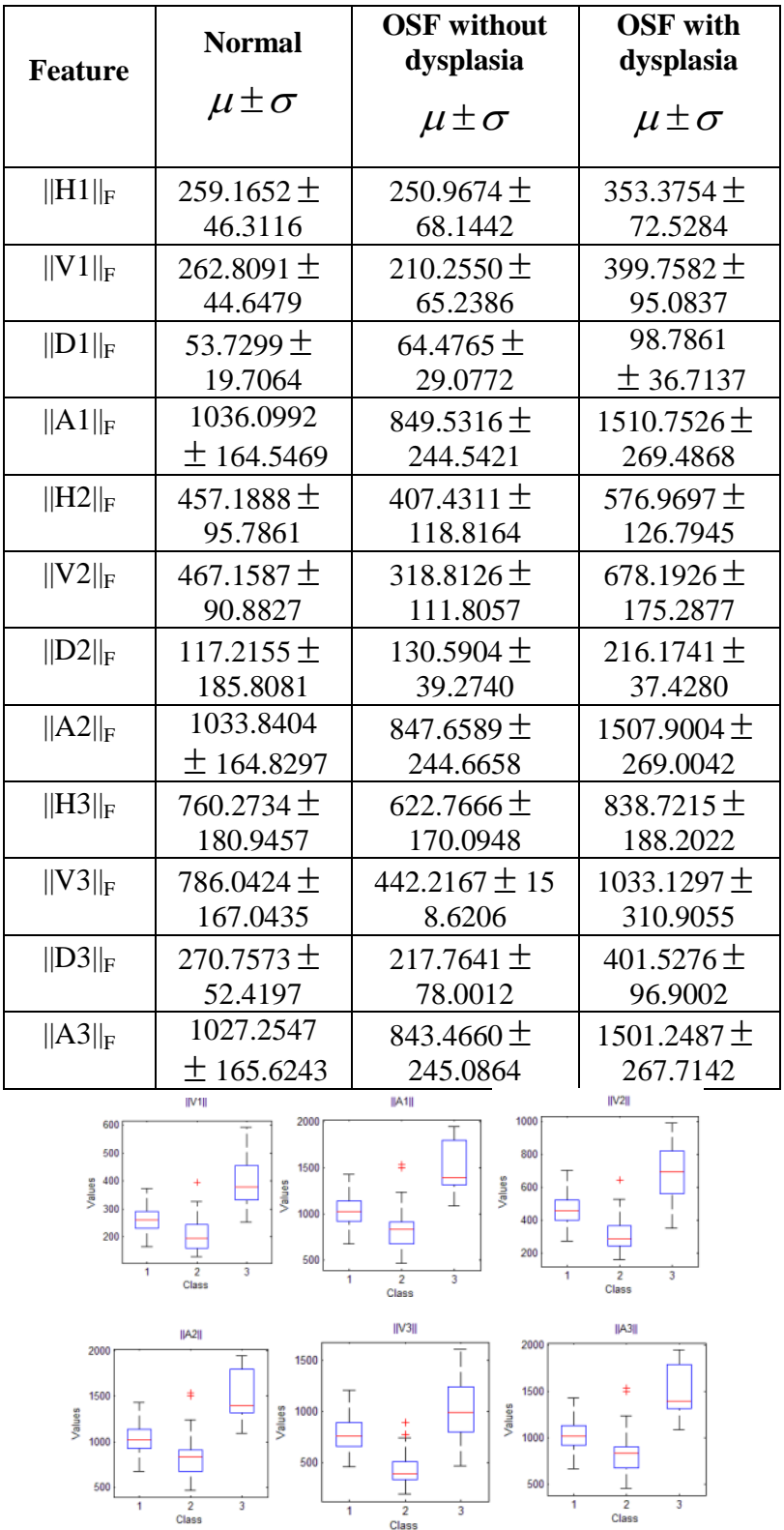

Figure 4: Boxplot of the selected features
One-way ANOVA has been done and the F-value and ranks of the features are shown in Table 2. The result of JB test is also shown in the same table and taking logical and of the result of both the tests; features numbered $\|\mathrm{V} 1\|_{\mathrm{F}},\|\mathrm{A} 1\|_{\mathrm{F}}$, $\|\mathrm{V} 2\|_{\mathrm{F}},\|\mathrm{A} 2\|_{\mathrm{F}},\|\mathrm{V} 3\|_{\mathrm{F}}$ and $\|\mathrm{A} 3\|_{\mathrm{F}}$ are selected for classification. The features denoted by $\|\mathrm{Ai}\|_{\mathrm{F}}$ are the approximate coefficients and $\|\mathrm{Hi}\|_{\mathrm{F}},\|\mathrm{Vi}\|_{\mathrm{F}}$ and $\|\mathrm{Di}\|_{\mathrm{F}}$ are the horizontal, vertical and diagonal component of detail coefficient respectively. Here ' $i$ ' denotes the step of decomposition. The confusion matrix for both the classifiers is obtained by averaging the output of each fold and is shown in Table 3.

Table 2. F-value, Rank and results of JB test of the Features

\begin{tabular}{|c|c|c|c|c|c|}
\hline \multirow{2}{*}{ Feature } & \multirow{2}{*}{ F-value } & \multirow{2}{*}{$\begin{array}{c}\text { Rank } \\
\text { of the } \\
\text { featur } \\
\text { e }\end{array}$} & \multicolumn{3}{|c|}{$\begin{array}{l}\text { Distribution of the } \\
\text { feature(JB test) }\end{array}$} \\
\hline & & & Class 1 & Class 2 & $\begin{array}{c}\text { Class } \\
3\end{array}$ \\
\hline$\|\mathrm{H} 1\|_{\mathrm{F}}$ & 28.352 & 9 & $\mathrm{~N}$ & $\mathrm{~N}$ & $\mathrm{~N}$ \\
\hline$\|\mathrm{V} 1\|_{\mathrm{F}}$ & 67.432 & 5 & $\mathrm{~N}$ & $\mathrm{~N}$ & $\mathrm{~N}$ \\
\hline$\|\mathrm{D} 1\|_{\mathrm{F}}$ & 26.440 & 10 & $\mathrm{~N}$ & $\mathrm{NN}$ & $\mathrm{NN}$ \\
\hline$\|\mathrm{A} 1\|_{\mathrm{F}}$ & 74.194 & 2 & $\mathrm{~N}$ & $\mathrm{~N}$ & $\mathrm{~N}$ \\
\hline$\|\mathrm{H} 2\|_{\mathrm{F}}$ & 18.478 & 11 & $\mathrm{NN}$ & $\mathrm{N}$ & $\mathrm{N}$ \\
\hline$\|\mathrm{V} 2\|_{\mathrm{F}}$ & 66.053 & 6 & $\mathrm{~N}$ & $\mathrm{~N}$ & $\mathrm{~N}$ \\
\hline$\|\mathrm{D} 2\|_{\mathrm{F}}$ & 112.041 & 1 & $\mathrm{NN}$ & $\mathrm{N}$ & $\mathrm{NN}$ \\
\hline$\|\mathrm{A} 2\|_{\mathrm{F}}$ & 73.973 & 3 & $\mathrm{~N}$ & $\mathrm{~N}$ & $\mathrm{~N}$ \\
\hline$\|\mathrm{H} 3\|_{\mathrm{F}}$ & 11.257 & 12 & $\mathrm{NN}$ & $\mathrm{N}$ & $\mathrm{N}$ \\
\hline$\|\mathrm{V} 3\|_{\mathrm{F}}$ & 62.123 & 7 & $\mathrm{~N}$ & $\mathrm{~N}$ & $\mathrm{~N}$ \\
\hline$\|\mathrm{D} 3\|_{\mathrm{F}}$ & 51.248 & 8 & $\mathrm{~N}$ & $\mathrm{~N}$ & NN \\
\hline$\|\mathrm{A} 3\|_{\mathrm{F}}$ & 73.496 & 4 & $\mathrm{~N}$ & $\mathrm{~N}$ & $\mathrm{~N}$ \\
\hline
\end{tabular}

$\mathrm{N}$ : Normal, NN: Non-normal, Class 1: Normal, Class 2: OSF without dysplasia, Class 3: OSF with dysplasia

Table 3. Confusion Matrix for Different Classifier

\begin{tabular}{|c|c|c|c|c|}
\hline \multirow[b]{2}{*}{ Classifier } & \multirow[b]{2}{*}{ Output } & \multicolumn{3}{|c|}{ Actual Class } \\
\hline & & Normal & $\begin{array}{l}\text { OSF } \\
\text { without } \\
\text { dysplasia }\end{array}$ & $\begin{array}{l}\text { OSF with } \\
\text { dysplasia }\end{array}$ \\
\hline \multirow{3}{*}{ Bayesian } & Normal & 15 & 0 & 1 \\
\hline & $\begin{array}{c}\text { OSF } \\
\text { without } \\
\text { dysplasia }\end{array}$ & 0 & 7 & 0 \\
\hline & $\begin{array}{l}\text { OSF with } \\
\text { dysplasia }\end{array}$ & 0 & 1 & 6 \\
\hline \multirow{3}{*}{ SVM } & Normal & 15 & 0 & 0 \\
\hline & $\begin{array}{c}\text { OSF } \\
\text { without } \\
\text { dysplasia }\end{array}$ & 0 & 7 & 0 \\
\hline & $\begin{array}{l}\text { OSF with } \\
\text { dysplasia }\end{array}$ & 0 & 1 & 7 \\
\hline
\end{tabular}


The comparison of the two classifiers is given in Table 4 . From the results of Table 4, it can be concluded that the selected features have potential to characterize the normal, OSF without dysplasia and OSF with dysplasia oral mucosa. SVM shows an overall accuracy of $96.67 \%$ whereas Bayesian classifier shows an overall accuracy of $93.33 \%$.

Table 4. Comparison of the two classifiers

\begin{tabular}{|c|c|c|c|c|}
\hline \multirow{2}{*}{ Classifier } & \multicolumn{3}{|c|}{ Individual Accuracy } & \multirow{2}{*}{$\begin{array}{c}\text { Overall } \\
\text { Accuracy }\end{array}$} \\
\cline { 2 - 5 } & Normal & $\begin{array}{c}\text { OSF } \\
\text { without } \\
\text { dysplasia }\end{array}$ & $\begin{array}{c}\text { OSF } \\
\text { with } \\
\text { dysplasia }\end{array}$ & \\
\hline Bayesian & 100 & 87.5 & 85.7 & 93.33 \\
\hline SVM & 100 & 87.5 & 100 & 96.67 \\
\hline
\end{tabular}

\section{CONCLUSIONS}

This paper facilitates the study of textural features of spinous layer of surface epithelium to characterize normal and different grades of precancerous oral mucosa. Bayesian and SVM based classification shows an overall accuracy of 93.33\% and $96.66 \%$ respectively which are comparable emphasizing that texture of spinous layer plays an important role in oral cancer diagnosis. The proposed scheme can be used to assist the oral pathologists to detect whether a test sample is normal or OSF without or with dysplasia. Hence a computer aided diagnostic (CAD) system for screening of precancerous oral mucosa can be developed in future.

\section{ACKNOWLEDGEMENTS}

The authors would like to thank Prof. R. R. Paul and Dr. M. Pal, GNDSIR, Kolkata, India for their clinical support and valuable advices.

\section{REFERENCES}

[1] Jadhav, A. S., Banerjee, S., Dutta, P. K., Paul, R. R., and Pal, M. 2006. Quantitative analysis of histopathological features of precancerous lesion and condition using image processing technique. 19th IEEE Int. Symposium on Computer-Based Medical Systems, 231-236.

[2] Mukherjee, A., Paul, R. R., Chaudhri, K., Chatterjee, J., Pal, M., and Banerjee, P. 2006. Performance analysis of different wavelet feature vectors in quantification of oral precancerous condition. Oral Oncology, 42, 914-928.

[3] Nanci, A. 2008. Oral Mucosa, Ten Cate's Oral Histology. Indian: Mosby- Elsevier.

[4] Pindborg J. J., and Sirsat, S. M. 1966. Oral submucous fibrosis, Oral Surg. Oral Med. Oral Pathol. 22, pp. 764779

[5] Cawson, R. A., and Odell, E. 2002. Cawson's Essentials of Oral Pathology and Oral Medicine. Churchill Livingstone.

[6] Landini, G., and Rippin, J. W. 1996. How important is tumour shape? Quantification of the epithelialconnective tissue interface in oral lesions using local connected fractal dimension analysis. The Journal of Pathology, 210-217.

[7] Landini, G., and Othman, I. E. 2004. Architectural analysis of oral cancer, dysplastic and normal epitheliam. Cytometry, 45-55.

[8] Abu-Eid, R., and Landini, G. 2006. Oral Epithelial Dysplasia: Can quantifiable morphological features help in the grading dilemma? First Image User and Developer Conference. Luxembourg.

[9] Kayser, K., Sandau, K., Bohm, G., Kunze, K., and Paul J, J. 1991. Analysis of soft tissue tumors by an attributed minimum spanning tree. Analytical \& Quantitative Cytology \& Histology, 329-334.

[10] Marcelpoil, R., Davoine, F., and Robert-Nicaud, M. 1994. Cellular sociology: parametrization of spatial relationships based on Voronoi diagram and Ulam trees. Fractals in biology and medicine, 201-209.

[11] Paul, R. R., Mukherjee, A., Dutta, P. K., Banerjee, S., Pal, M., Chatterjee, J., Mukhopadhyay, K, and Chaudhuri, K.2005. A novel wavelet neural network based pathological stage detection technique for an oral precancerous condition. Journal of Clinical Pathology, 58, 932-938.

[12] Mukherjee, A., Paul, R. R., Chaudhri, K., Chatterjee, J., Pal, M., and Banerjee, P. 2006. Performance analysis of different wavelet feature vectors in quantification of oral precancerous condition. Oral Oncology, 42, 914 - 928.

[13] M. M.R.K., Pal, M., Bomminayuni, S.K., Chakraborty, C., Paul, R.R., Chatterjee, J., Ray, A.K. 2009. Automated classification of cells in sub-epithelial connective tissue of oral sub-mucous fibrosis-An SVM based approach, Computers in Biology and Medicine, 39(12), 1096-1104.

[14] Muthu Rama Krishnan, M., Shah, P., Pal, M., Chakraborty, C., Paul, R.R., Chatterjee, J., Ray, A.K. 2010. Structural markers for normal oral mucosa and oral sub-mucous fibrosis. Micron 41 (4), 312-320.

[15] Perona, P., and Malik, J. 1990. Scale-space and edge detection using anisotropic diffusion. IEEE Trans. on Pattern Analysis and Machine Intelligence, 12 (7).

[16] Montagna W, Parakkal PF. 1974. The Structure and Function of Skin, 3rd edn. New York: Academic Press.

[17] Mallat, S. 1989. A theory for multiresolution signal decomposition: the wavelet representation, IEEE transaction on Pattern Analysis and machine intelligence. 11(7), pp 674-693.

[18] Gun, A. M., Gupta, M. K., Dasgupta, B. 2008 Fundamentals of Statistics. Vol. 2. World Press.

[19] Jarque, C. M., Bera, A. K. 1987. A test for normality of observations and regression residuals. International Statistical Review, 55(2), 163-172.

[20] Duda, R., Hart, P., \& Stork, D. 2007. Pattern classification. Wiley India.

[21] Vapnik, V. 1998. Statistical learning theory. New york: Wiley.

[22] Gunn, S. R. 1998. Support Vector Machines for Classification and Regression. 